experienced prescribers, as well as the encouragement from nurse leaders to be confident prescribers themselves and enhance patient care (Wilson, et al.,2014).

At St Christopher's, we have encouraged our specialist nurses to undertake academic training to become independent nurse prescribers, and have seen practice flourish. A recent audit of anticipatory prescribing demonstrated the value of nurse prescribers in hospice care, in that $55 \%$ of all anticipatory prescribing was undertaken by nurse independent prescribers. This highlights the pressing need for continuing professional development.

Until 2020, St Christopher's supported our own nurses to attend a national study day to provide pharmacology and prescribing updates, but with the assault of COVID-19, we needed to think differently about how to deliver this. In doing so, we developed a monthly online community of practice which has grown in popularity. Joining this we have over 100 nurses working in palliative and end-of -life care who are non-medical prescribers. Once a month, using video conferencing technology, members of the community join us from all the four nations of the UK, to learn together, share knowledge and access the expertise of invited speakers.

This presentation will outline the significant role of nurse prescribers within the hospice setting, and highlight the need to ensure the availability of CPD. In doing so, we will highlight one such approach to providing $\mathrm{CPD}$, along with raising the national profile of this important work.

\section{P-172 MAINTAINING THE CONFIDENCE OF NON-MEDICAL PRESCRIBERS - BENEFITS OF A VIRTUAL NMP FORUM}

${ }^{1}$ Jo Poultney, ${ }^{2}$ Karen Badgery. ${ }^{1}$ South Warwickshire Foundation Trust, Warwick, UK; ${ }^{2}$ Myton Hospice, Coventry, UK

\subsection{6/spcare-2021-Hospice.188}

Background An increasing number of specialist palliative care nurses are embarking on training to become a prescriber. Non-medical prescribers (NMPs) play a very important role in supporting patients and allowing a more efficient and responsive service (Cope, Abuzour, Tully, 2016). Despite the training being rigorous, one in 10 prescribers say they lack confidence in their new skill (Nurse prescribers competent but not confident. Nursing Standard. 20, 30, 10-10. doi: 10.7748/ns.20.30.10.s15). If appropriate support is not provided to this group of professionals, benefit of their training may be lost. In 2016 a monthly NMP forum was set up in Coventry and Warwickshire to provide a supportive environment to share experiences. Ten nurses attended on average each month, supported by a consultant and pharmacist.

At the beginning of the pandemic it was decided to offer the opportunity to meet via Teams. 88 NMPs have now requested to join the invite list. On average between 25 and 30 attend each meeting. Being on Teams has enabled far more people to be able to join from across the county. A survey was carried out to understand more about the group and to ensure that it was meeting the needs of those who attend.

Results People who attend vary between being a prescriber for $>10$ years to those just embarking on their training. Having members of other specialist palliative care teams in different areas allows those who attend to see similarities/differences in working practice and take best practice back to their area. They report valuing the opportunity to discuss complex symptoms and to share different ways to manage them. It is seen as a 'safe place' where they can receive support, knowledge, education and supervision to support their role as an NMP. $85 \%$ report that the meeting meets their learning needs. $70.3 \%$ more likely to attend if meeting continues to be held virtually

Conclusion The results from the survey have reinforced the plan to continue offering these sessions in their current format.

\section{P-173 AN ICS WIDE VIRTUAL NON-MEDICAL PRESCRIBER FORUM: A PILOT PROPOSAL}

Abi Ponnampalam. Farleigh Hospice, Chelmsford, UK

\subsection{6/spcare-2021-Hospice. 189}

Background Non-medical prescribers (NMPs) have a significant role to play in providing effective and timely access to medications for patients at the end-of-life (Ziegler, Bennett, Mulvey et al., 2018; Weeks, George, Maclure, et al., 2016). There is increasing recognition of the positive contribution of NMPs for patient care especially during out-of-hours periods (Webb \& Gibson, 2011).

Worryingly, not all NMPs feel prepared to prescribe after qualifying with some choosing not to at all (Ziegler, Bennett, Blenkinsopp, et al., 2015; Ryan-Woolley, McHugh, Luker, 2007). Prescribing decision making can be perceived as complex with recent research supporting the role of peer mentoring and support in developing professional practice (Latham \& Nyatanga, 2018; Bowskill, Meade, Lymn, 2014).

Peer support has been shown to improve NMP confidence as well as positively influence prescribing (Otway, 2013; McIntosh, Stewart, Forbes-McKay et al., 2016). As palliative care services adapt to the challenges of the pandemic, virtual forums offer a platform for peer support and cross-organisational learning without the limitations of in-person meetings (Dunleavy, Preston, Bajwah, et al., 2021).

We propose a virtual NMP forum based on Lave and Wenger's 'Community of Practice', a social learning theory where knowledge develops collectively through collaboration within a group they enhance their skills and confidence in their roles as NMPs. By opening up the forum to specialist palliative care NMPs working across organisations and care settings, we hope to foster closer professional relationships and support innovation with the aim of developing services to meet the needs of the local community.

Aim To pilot and evaluate a virtual NMP Forum based on a 'Community of Practice' model across a newly designated Integrated Care System spanning three independent hospices, community and acute trust providers of specialist palliative care services.

Methods Bi-monthly virtual meetings will be held to provide opportunities for peer support, sharing best practice and networking across organisations and care settings. The session design will be fluid with opportunity to modify based on NMP feedback. Sessions will be evaluated for relevance to their role as NMPs, ease of attendance and peer support.

We hope to successfully pilot and incorporate this model within the region. 